\title{
Role of Preprocedural Computed Tomography in Transcatheter Aortic Valve Implantation
}

\section{Stellenwert der präprozeduralen Computertomografie bei kathetergestützten Aortenklappenimplantationen}

\author{
Authors \\ L. Lehmkuhl' ${ }^{1}$ B. Foldyna ${ }^{1}$, M. Haensig ${ }^{2}$, K. von Aspern ${ }^{2}$, C. Lücke ${ }^{1}$, C. Andres ${ }^{1}$, M. Grothoff ${ }^{1}$, F. Riese ${ }^{1}$, S. Nitzsche ${ }^{1}$, \\ D. Holzhey², A. Linke ${ }^{3}$, F.-W. Mohr ${ }^{2}$, M. Gutberlet ${ }^{1}$ \\ Affiliations \\ 1 Dept. of Interventional and Diagnostic Radiology, University of Leipzig - Heart Center, Leipzig \\ Clinic for Cardiac Surgery, University of Leipzig - Heart Center, Leipzig \\ Dept. of Cardiology, University of Leipzig - Heart Center, Leipzig
}

Key words
aorta
aortic valve
heart
CT
percutaneous

eingereicht 25.4 .2013 akzeptiert $\quad 12.7 .2013$

\section{Bibliography}

DOI http://dx.doi.org/

10.1055/s-0033-1350416

Published online: 2.9 .2013

Fortschr Röntgenstr 2013; 185 :

941-949 @ Georg Thieme

Verlag KG Stuttgart · New York ·

ISSN 1438-9029

\author{
Correspondence \\ Dr. Lukas Lehmkuhl \\ Diagnostische und \\ Interventionelle Radiologie, \\ Universität Leipzig - \\ Herzzentrum \\ Strümpell Str. 38 \\ 04289 Leipzig \\ Tel.: ++49/341/8651702 \\ Fax: ++49/341/8651803 \\ lukas.lehmkuhl@med.uni- \\ leipzig.de
}

\section{Abstract \\ $\nabla$}

Transcatheter aortic valve implantation (TAVI) is currently considered an acceptable alternative for the treatment of patients with severe aortic stenosis and a high perioperative risk or a contraindication for open surgery. The benefit of TAVI significantly outweighs the risk of the procedure in patients requiring treatment that are not suitable for open surgery, and leads to a lower mortality in the one-year follow-up. The absence of a direct view of the aortic root and valve remains a challenge for the transcatheter approach. While direct inspection of the aortic valve during open surgery allows an adequate prosthesis choice, it is crucial for TAVI to know the individual anatomical details prior to the procedure in order to assure adequate planning of the procedure and proper prosthesis choice and patient selection. Among the imaging modalities available for the evaluation of patients prior to TAVI, computed tomography (CT) plays a central role in patient selection. CT reliably visualizes the dimensions of the aortic root and allows a proper choice of the prosthesis size. The morphology of the access path and relevant comorbidities can be assessed. The present review summarizes the current state of knowledge regarding the value of $\mathrm{CT}$ in the evaluation of patients prior to TAVI.

Key Points:

- CT plays a central role in patient selection and planning prior to TAVI.

- CT reliably detects the dimensions of the aortic root including the size of the aortic annulus, the degree of valve calcification and the morphology of the access routes.

- CT provides a more accurate measurement of the aortic annulus than 2D TEE and CT is the only imaging modality that allows a risk assessment for paravalvular leakages based on the calcification of the aortic valve.

Citation Format:

- Lehmkuhl L, Foldyna B, Haensig M etal. Role of Preprocedural Computed Tomography in Transcatheter Aortic Valve Implantation. Fortschr Röntgenstr 2013; 185: 941-949

\section{Zusammenfassung \\ $\nabla$}

Katheterbasierte Aortenklappenimplantationen (TAVI) gelten mittlerweile als akzeptierte Alternative zur Behandlung von Patienten mit hochgradiger Aortenklappenstenose und hohem perioperativem Risiko oder einer Kontraindikation für einen offen-chirurgischen Aortenklappenersatz (AKE). Der Nutzen der TAVI überwiegt das Risiko des Eingriffs deutlich bei behandlungsbedürftigen Patienten, die nicht für einen offenchirurgischen AKE geeignet sind, und führt zu einem Absinken der Mortalität im 1-Jahres-Follow-up. Eine Herausforderung bei der kathetergestützten Vorgehensweise stellt das Fehlen einer direkten Sicht auf die Aortenwurzel und -klappe während der Implantation dar. Während beim offen-chirurgischen AKE die Aortenklappe direkt inspiziert und eine Prothesenwahl getroffen werden kann, ist es bei der TAVI erforderlich, die individuellen anatomischen Details vor der Prozedur zu kennen, um eine adäquate Planung der Prozedur, eine geeignete Prothesenwahl und Patientenselektion zu ermöglichen. Unter den für eine Evaluation von Patienten vor TAVI verfügbaren bildgebenden Modalitäten nimmt die Computertomografie (CT) mittlerweile eine zentrale Rolle bei der Patientenselektion ein, da mit ihr zuverlässig die Dimensionen der Aortenwurzel einschließlich der Wahl der Prothesengröße, die Morphologie des Zugangswegs und relevante Komorbiditäten erfasst werden können. Die vor- 
liegende Übersichtsarbeit fasst den aktuellen Stand des Wissens zum Stellenwert der CT bei der Evaluation von Patienten vor TAVI zusammen.

\section{Introduction}

Transcatheter aortic valve implantation (TAVI), which was performed for the first time in a human in 2002, is currently considered an acceptable alternative for treating patients with severe aortic stenosis (AS) and a high perioperative risk or a contraindication for open surgical aortic valve replacement $[1,2]$.

AS is the most common heart valve disease in the Western world and primarily occurs at an advanced age [3]. Valve replacement is generally indicated when the stenosis is symptomatic $[2,4]$. While open surgical aortic valve replacement is a low-risk procedure and is considered the gold standard, it is contraindicated in up to $30 \%$ of older patients with symptomatic AS [5]. The reason for this is the increased perioperative risk due to comorbidities such as a reduced left ventricular ejection fraction, renal dysfunction, or a chronic obstructive pulmonary disease [5]. However, if left untreated, symptomatic AS has a poor prognosis with an annual mortality rate of $25 \%$ [6].

The benefits of TAVI significantly outweigh the risk of the procedure in patients requiring treatment that are not suitable for open surgical aortic valve replacement and leads to a reduction of the mortality in the one-year follow-up of approximately $50 \%$ [7]. In patients with an increased perioperative risk, it was able to be shown that TAVI is not inferior to open surgical aortic valve replacement with respect to mortality [8].

The spectrum of complications during TAVI differs from those of open surgical aortic valve replacement. Cerebral infarctions and method-associated complications, such as vascular damage or bleeding, occurred more frequently $[7,8]$. Moreover, paravalvular leaks, which are associated with a higher mortality rate when severe, were frequently observed [9]. The current state of knowledge regarding TAVI procedures is comprehensively summarized in a consensus article by large American and European professional associations [2].
A challenge in the catheter-assisted procedure is the lack of a direct view of the aortic root and valve during implantation. While the aortic valve can be directly inspected and a prosthesis can be selected during open surgical aortic valve replacement, it is necessary in the case of TAVI to know the individual anatomical details prior to the procedure to allow adequate planning of the procedure and suitable prosthesis and patient selection.

Suitable and frequently used preprocedural imaging modalities are transesophageal echocardiography (TEE) and multi-row computed tomography (CT), while magnetic resonance imaging (MRI) is used less frequently $[10,11]$. CT is routinely performed in many large centers to evaluate patients prior to TAVI $[12,13]$. A further indication of the increasing importance of $\mathrm{CT}$ is a recently published consensus document by the Society of Cardiovascular Computed Tomography (SCCT; [14]). Fluoroscopy, TEE, and sometimes $3 \mathrm{D}$ rotation angiography are primarily used intraprocedurally $[2,15]$.

\section{Available prostheses and implantation techniques $\nabla$}

The most important prostheses that are currently available for TAVI and have been primarily used for this procedure to date are the self-expanding Medtronic CoreValve ${ }^{\mathrm{TM}}$ (Medtronic, Minneapolis, MN, USA) and the balloon-expandable Edwards SAPIEN ${ }^{\mathrm{TM}}$ (Edwards Lifescience, Irvine, CA, USA) ( $\bullet$ Fig. 1). There are additional prostheses types which in some cases have only been used in studies thus far [16]. The Medtronic CoreValve ${ }^{\mathrm{TM}}$ prosthesis is comprised of a nitinol frame that supports a tricuspid valve made of porcine pericardial tissue. The middle of the prosthesis is tapered, and the valve of the prosthesis has a slight supra-annular position. The prosthesis is available is sizes $23 \mathrm{~mm}, 26 \mathrm{~mm}$, $29 \mathrm{~mm}$, and $31 \mathrm{~mm}$ and is typically implanted transfemorally or alternatively via the subclavian artery in exceptional cases. This requires an $18 \mathrm{~F}$ delivery system $[14,17]$.

Depending on the version, the Edwards SAPIEN ${ }^{\mathrm{TM}}$ prosthesis is comprised of a cylindrical stainless steel or cobalt-chromium frame which supports a tricuspid valve made of bovine pericardial tissue. The lower two-thirds of the frame and the stent graft are covered, while the upper third is not a

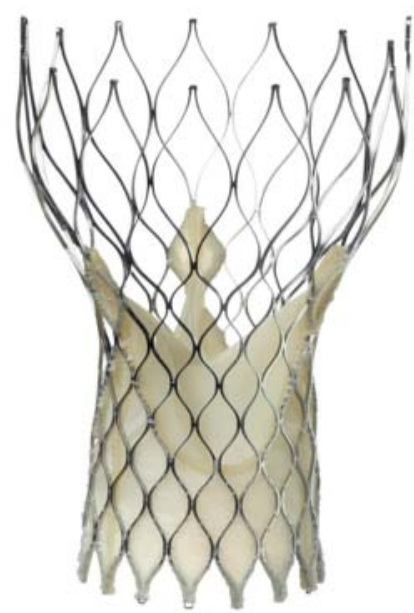

b

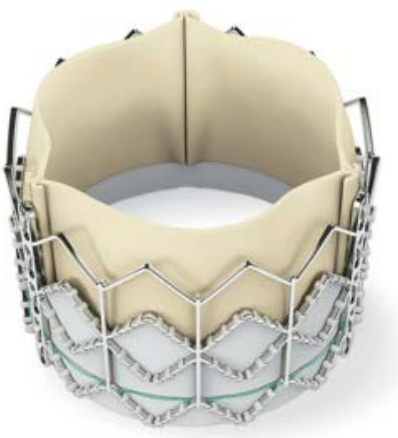

Fig. 1 a Medtronic CoreValve ${ }^{\mathrm{TM}}$ (Medtronic, Minneapolis, MN, USA). The prosthesis has a narrowed shape at the height of the aortic sinus and coronary ostia. b Edwards SAPIEN ${ }^{\mathrm{TM}}$ (Edwards Lifescience, Irvine, CA, USA). This prosthesis has a cylindrical shape. The lower two-thirds of the frame are covered and placed into the aortic annulus while the upper third without cover is placed into the aortic sinus. 

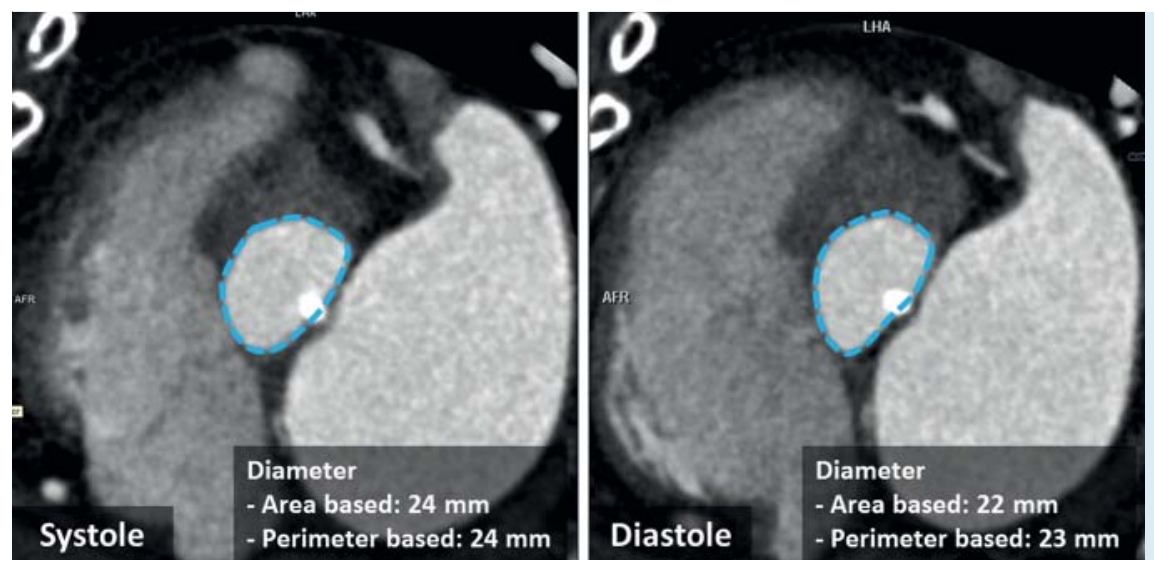

Fig. 2 Deformation of the ovaloid aortic annulus shape during the cardiac cycle. The aortic annulus has the largest area during systole and shows an asymmetrical deformation during diastole.
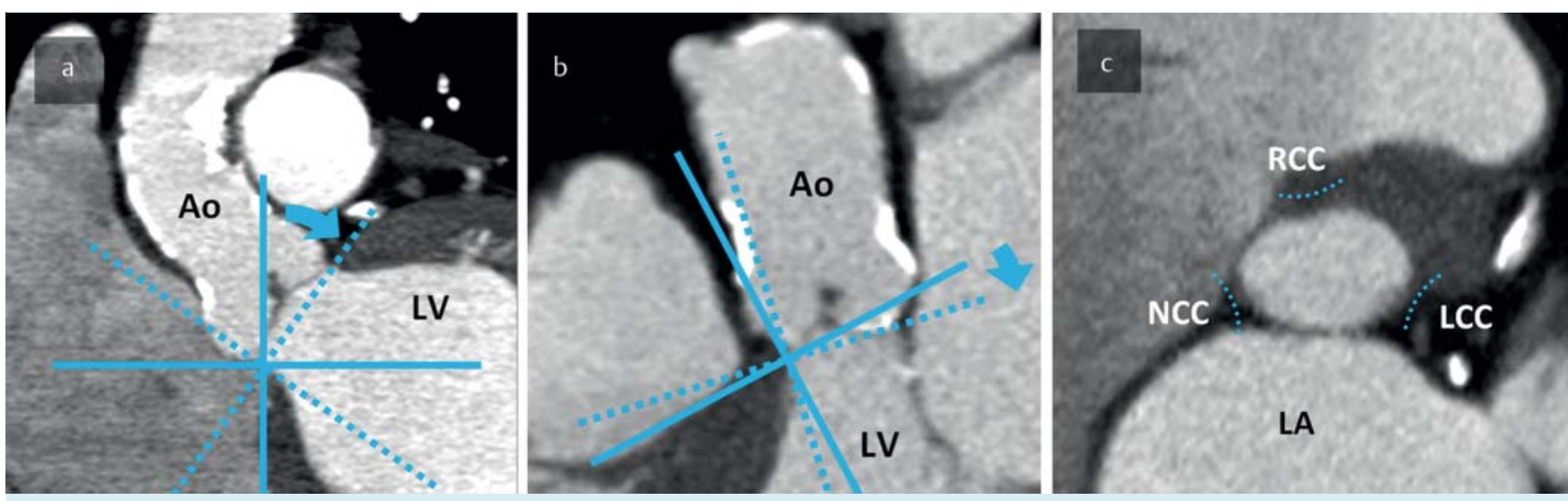

Fig. 3 Planning a multiplanar reconstruction through the aortic annulus: a coronal view; a plane is defined by the deepest insertions of at least two aortic valve cusps. b corresponding sagittal view; adaptation of the plane orientation to the deepest insertion of the third cusp. $\mathbf{c}$ The resulting third view now shows the oval aortic annulus and can be used for measurement. $\mathrm{Ao}=$ aorta, $\mathrm{LV}=$ left ventricle; $\mathrm{RCC} / \mathrm{LCC} / \mathrm{NCC}=$ right coronary/left coronary $/$ non-coronary cusp, $\mathrm{LA}=$ left atrium. covered. The prosthesis is placed with the covered portion in the aortic annulus while the uncovered part extends into the aortic bulb. The following sizes are available: $23 \mathrm{~mm}, 26 \mathrm{~mm}$, and $29 \mathrm{~mm}$. The preferred implantation paths are transfemoral and transapical. $16 \mathrm{~F}$ or $18 \mathrm{~F}$ delivery systems are used for the transfemoral implantation route and $24 \mathrm{~F}$ or $26 \mathrm{~F}$ systems for the transapical implantation route. The version based on a cobalt-chromium frame can be introduced with a smaller $18 \mathrm{~F}$ delivery system $[14,18]$.

In addition to the transfemoral and transapical implantation routes and implantation via the subclavian artery when using small delivery systems, a transaortic implantation route via the ascending aorta was also described [19]. During implantation, the prostheses are anchored in the aortic annulus and the native aortic valve cusps are pressed against the wall of the aortic bulb.

\section{Preprocedural computed tomography \\ $\nabla$}

While severe AS is typically diagnosed via echocardiography and Doppler in accordance with the current guidelines starting at an aortic valve orifice area $<1 \mathrm{~cm}^{2}\left(<0.6 \mathrm{~cm}^{2} / \mathrm{m}^{2}\right)$ or an average gradient $>40-50 \mathrm{mmHg}$ [24-26], the suitability of a patient for TAVI and the planning of the procedure are largely derived from preprocedural imaging [2].
There are currently no established recommendations for imaging in the case of TAVI evaluation, resulting in significant variability in imaging protocols depending on the experience of the particular medical facility.

\section{Aortic root}

\section{Measuring the aortic annulus}

The measurement of the aortic annulus is one of the most important steps in the evaluation prior to TAVI and is the basis for the selection of the prosthesis size.

The aortic annulus as the landing zone for prosthesis implantation cannot be directly visualized. The aortic annulus is defined as a virtual ring running on one plane through the basal insertions of the aortic valve cusps [20,21]. It has an oval shape to varying degrees and changes shape during the cardiac cycle even in patients with severe AS and significant valve calcifications ( $\bullet$ Fig. 2) $[22,23]$.

While the size of the aortic annulus was initially determined almost exclusively via 2-dimensional TEE, numerous studies have since proven that the individually varying, dynamic, and complex 3-dimensional geometry of the aortic annulus cannot be reliably visualized with $2 \mathrm{D}$ imaging and can only be sufficiently displayed by $3 \mathrm{D}$ imaging $[23,27-$ 29]. A prosthesis selection solely on the basis of a $2 \mathrm{D}$ TEE measurement of the aortic annulus can underestimate the actual size $[23,28]$ and is associated with an increased rate 

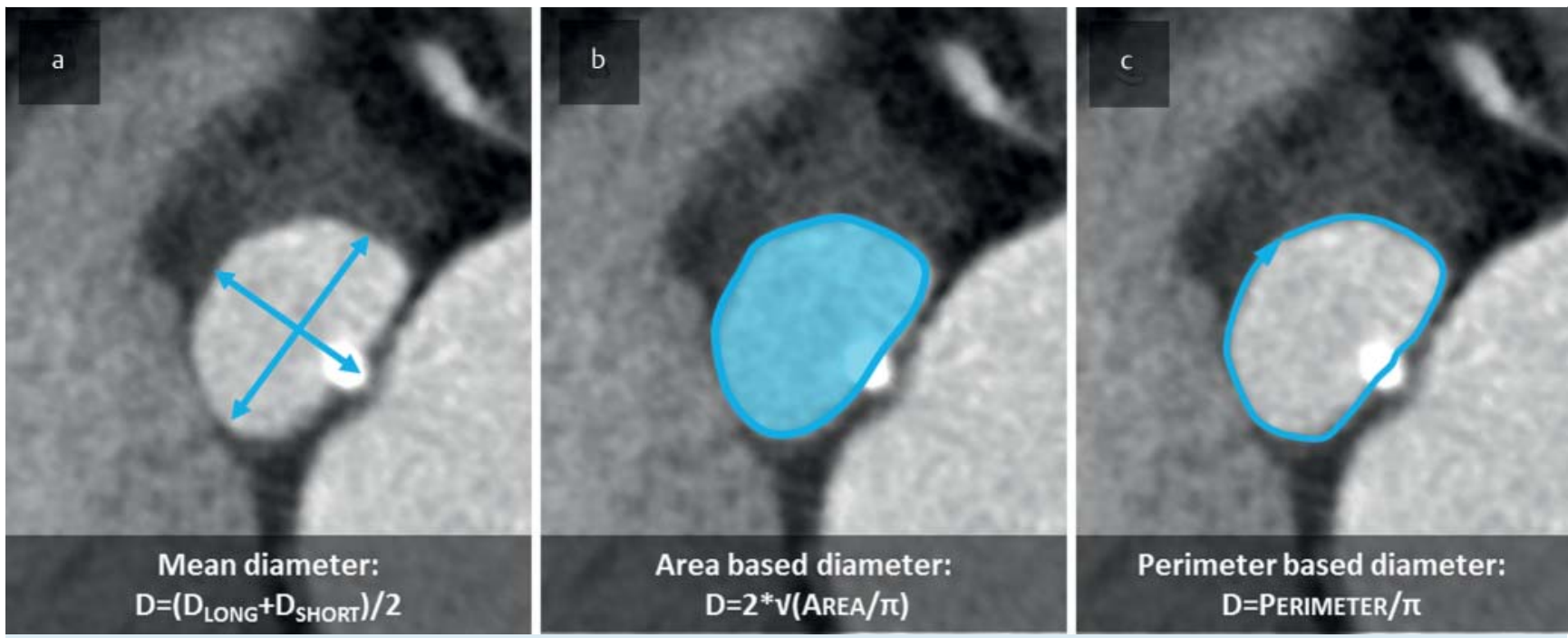

Fig. 4 Different methods of measurement to determine the annulus diameter: $\mathbf{a}$ average diameter of long and short diameter, $\mathbf{b}$ area-based and $\mathbf{c}$ perimeterbased diameter: The effective diameter corresponds to a virtual circle with the same area and the same perimeter.
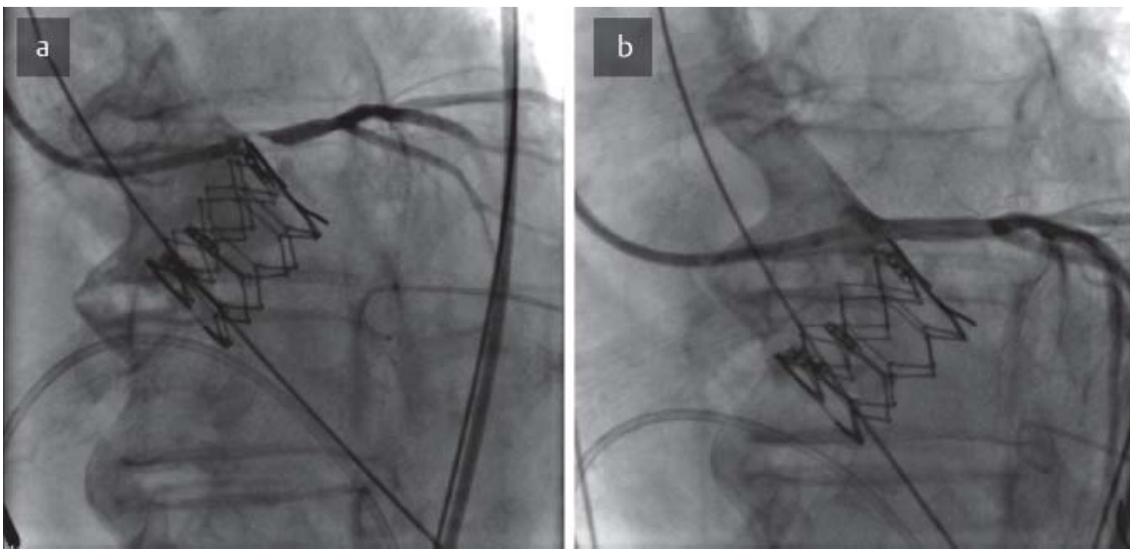

Fig. 5 a Occlusion of the left main artery during transfemoral implantation of an Edwards SAPIEN ${ }^{\mathrm{TM}}$ prosthesis. The coronary ostia is exceeded only by the non-covered part of the prosthesis, so not the prosthesis itself, but displaced cusp material is likely to be the reason for the occlusion of the ostium. Note the tubular shape of the aortic sinus, which offers little space to accommodate the displaced cusps. b Successful re-opening of the left main artery.

of paravalvular leaks [7, 8]. Moreover, the selection of the prosthesis size differed in further studies on the basis of the imaging modality used $[23,30,31]$. Performing measurements during the procedure via $3 \mathrm{D}$ rotation angiography is also not recommended since a high interobserver variability compared to CT was described [15] and the size of the aortic annulus would no longer be available as a parameter for preprocedural patient selection.

For the measurement it is necessary to create a multiplanar reconstruction on the basis of thin-slice images and to place a plane through the 3 lowest insertion points of the aortic valve cusps. The aortic annulus is typically oval on this plane ( $\bullet$ Fig. 3 ).

It is recommended to derive the diameter of the aortic annulus as the so-called effective diameter of the cross-sectional area $(D=2 * \sqrt{(\text { area } / \pi)})$ or the perimeter $(D=$ perimeter $/ \pi)$ since these dimensions also provide reliable values in the case of differences in the shape of the aortic annulus [23]. In addition, an average diameter can be calculated from the separately measured short and long diameters. Since the aortic annulus has the largest cross-sectional area in systole ( $30 \%$ RR interval), this cardiac phase should be used for measuring the area-based effective diameter
$[14,23]$. An effective diameter derived from the perimeter is presumably less susceptible to the change in the crosssectional area during the cardiac cycle [32] ( $\bullet$ Fig.4).

Relationship between coronary ostia, aortic sinus, and aortic valve cusp length

The complex 3-dimensional structure of the aortic sinus is usually onion-shaped but can have a tubular shape in some patients. In these patients there is less or insufficient space for the native aortic valve cusps that are displaced to the sinus wall by the prosthesis, thus potentially resulting in coronary obstructions with a potentially fatal outcome (० Fig. 5).

The position of the coronary ostia can also vary. While the coronary ostia are at sufficient distance from the aortic annulus for prosthesis implantation in most patients, some patients have low coronary ostial heights with a distance of only several millimeters from the aortic annulus. This also poses a potential risk for coronary obstructions ( $\bullet$ Fig. 6 ). Coronary anomalies with an atypical position of the ostia can also present a problem.

The average distances of the coronary ostia are in the range of $12-16 \mathrm{~mm}$ for the right coronary artery and $12-17 \mathrm{~mm}$ 

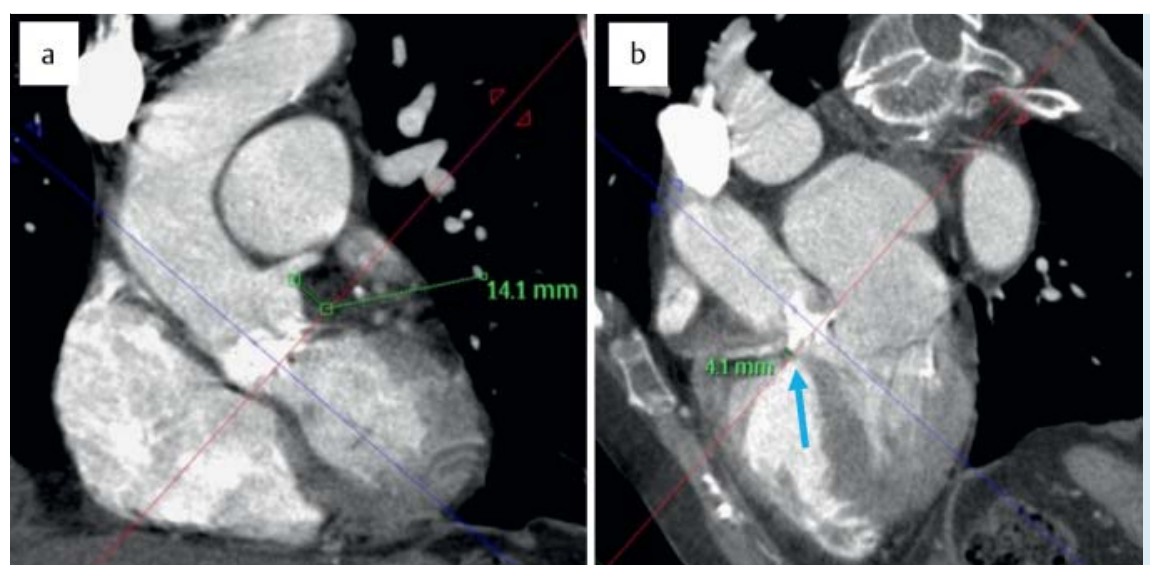

Fig. 6 Variability of the distances between the aortic annulus and the coronary ostia in an 81-yearold woman prior to TAVI. a Inconspicuous ostial height of the left coronary artery with $14.1 \mathrm{~mm}$. b Low ostial height of the right coronary ostium with $4.1 \mathrm{~mm}$. Hence, the patient received an open surgical aortic valve replacement. The low ostial height was confirmed intraoperatively.
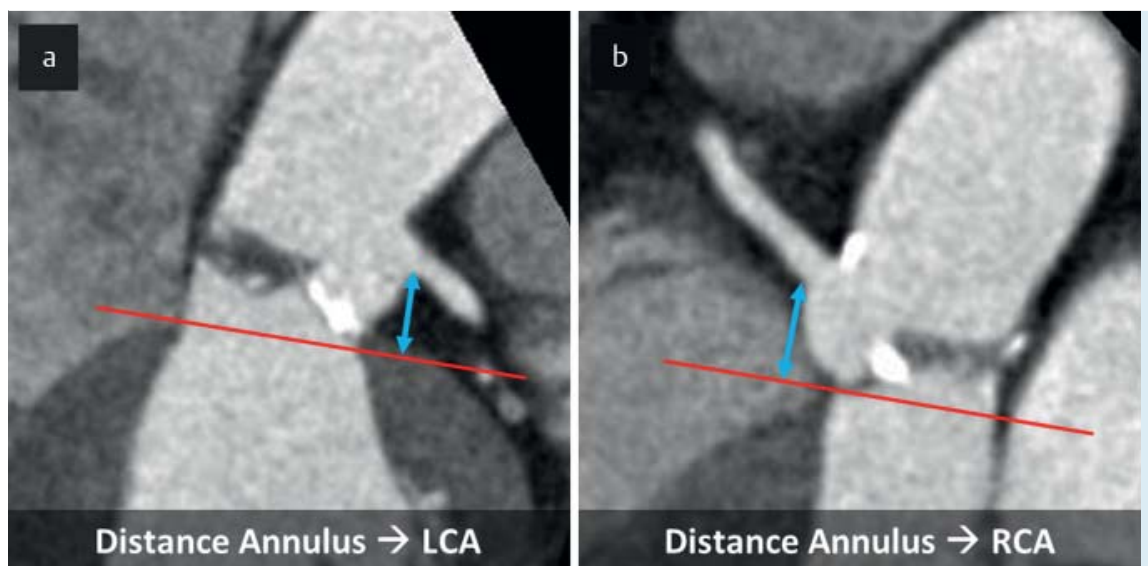

Fig. 7 In order to measure the left coronary a and right coronary b ostial height, the distance from the inferior part of the coronary ostium to the aortic annulus plane can be measured perpendicularly.

Distance Annulus $\rightarrow$ RCA

for the left coronary artery $[23,27]$. However, this can vary in both coronary arteries with very short distances of only a few millimeters [23]. The risk of a coronary obstruction presumably depends on the relationship between ostial distance, aortic sinus size, and aortic valve cusp length. If the implanted prosthesis presses the native aortic valve cusps into the coronary sinus, a large sinus, high coronary ostia, and short aortic valve cusps are associated with a low risk of coronary obstruction while long aortic valve cusps, short ostial distances, and a tubular sinus are associated with a high risk. However, the risk resulting from these three anatomical parameters has not yet been conclusively studied. In general, it is assumed that the risk for a coronary obstruction when using the Medtronic CoreValve ${ }^{\mathrm{TM}}$ prosthesis is low due to the tapered shape which takes up less space on the level of the coronary sinus, while a minimal ostial distance of $10-14 \mathrm{~mm}$ is recommended for the cylindrical Edwards SAPIEN ${ }^{\mathrm{TM}}$ prosthesis $[14,33]$.

While it is difficult and often unreliable to measure ostial distances using 2 D TEE, CT is highly suitable for this purpose due to the ability to use multiplanar reconstructions from $3 \mathrm{D}$ data. In this case, the distance from the inferior edge of the coronary ostium to the aortic annulus plane can be measured perpendicularly ( $\bullet$ Fig. 7 ).

In the case of tricuspid valves, the aortic sinus is not round and can be described either as the maximum diameter or by three individual diameters from a coronary sinus to the opposite intercommissural part of the sinus. The left and right aortic valve cusps can be visualized most reliably in diastole
(70\% RR interval) and characterized by a polygon-based distance measurement ( $\bullet$ Fig. 8 ). If the distance between the cusp insertion and the left/right coronary artery is subtracted from the aortic valve cusp length, the potential degree of overlapping for a coronary ostium at maximum lateral displacement of the cusp can be estimated on the basis of the difference. However, the clinical importance of a potential degree of overlapping has not yet been substantiated by studies.

\section{Degree of aortic valve calcification}

In the case of AS, the aortic valve usually exhibits significant or excessive calcification ( $\bullet$ Fig.9). While the cusps and annular calcifications are removed during open surgical aortic valve replacement, the calcifications are pressed to the side into the aortic sinus by the prosthesis in the case of TAVI. In the case of significant calcification of the cusps, this increases the risk for coronary obstructions $[27,34]$. There is also a risk of paravalvular leaks since the calcifications prevent the prosthesis from fully unfolding and from properly resting against the wall of the aortic annulus [35 - 38]. In a study including 120 patients who all received an Edwards SAPIEN ${ }^{\mathrm{TM}}$ prosthesis via transapical TAVI, we were able to show that the degree of calcification is a predictor for relevant postprocedural, paravalvular leaks [36]. Consideration of the degree of calcification when determining indication is thus recommended since relevant paravalvular leaks are associated with a significantly higher mortality rate [9]. While the degree of calcification was determined in our study via a me- 

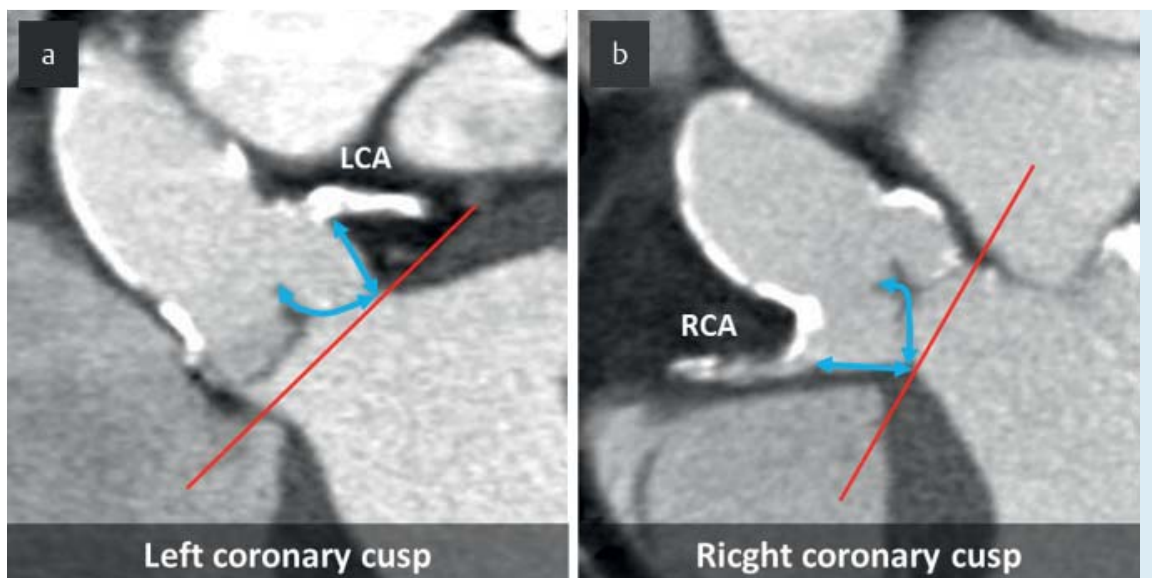

Fig. 8 Measuring the length of the left coronary a and right coronary $\mathbf{b}$ cusp. By subtracting the distance between the left (LCA)/right (RCA) coronary ostium and the corresponding basal cusp insertion from the cusp length, the potential degree of overlap can be estimated.
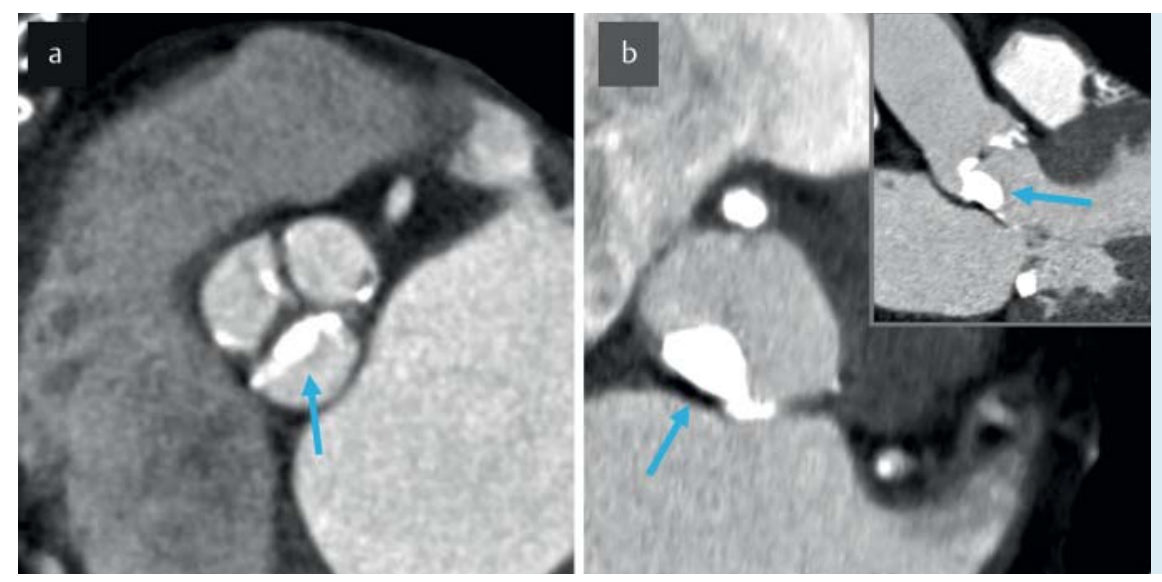

Fig. 9 a Asymmetric calcification of the valve cusps. b Eccentric calcification of the non-coronary cusp, which protrudes into the left ventricular outflow tract and makes a complete unfolding of the prosthesis impossible. Because of this finding, the patient was not considered for TAVI.

thod derived from the Agatston score for coronary arteries [39] on the basis of a separate native scan, a standardized method for describing the degree of calcification has not yet been established in the clinical routine. For the sake of orientation, a probability for the occurrence of a relevant paravalvular leak, which is for example $20 \%$ with a valve calcification score of approx. 2000 and $50 \%$ with a score of approx. 3700 , can be derived on the basis of our study data Therefore, the degree of calcification should first be evaluated visually on the basis of contrast-enhanced scans.

\section{Angulation of the aortic annulus plane}

Before a prosthesis is implanted in the aortic annulus, it is necessary to verify that the prosthesis is in correct alignment in relation to the aortic annulus. For this purpose, the projection is aligned by means of aortography via a catheter in the ascending aorta so that the three lowest points of the cusps are on one plane and the right coronary cusp is projecting exactly between the left coronary cusp and the non-coronary cusp. If the prosthesis is aligned parallel to the fluoroscopic beam path so that the edges of the prosthesis opening project over one another and the prosthesis opening is not visible, it can be assumed that the prosthesis is parallel to the aortic annulus.

If the patient is positioned with almost the same alignment during the TAVI procedure as during preprocedural CT, the same angle used in CT can be used for setting the fluoroscopy projection to be parallel to the aortic vale. As described above, the aortic annulus plane is set as a multiplanar reconstruction and the angle of a plane that is perpendicular to the aortic annulus and runs through the center of the right coronary sinus and the opposite intercommissural part of the sinus is measured. As a result, the projection angle can be reliably predicted, aortography for aligning fluoroscopy can be eliminated, and the procedure duration and radiation exposure can be lowered [40-42].

\section{Aorta and iliofemoral vessels}

Vascular complications during TAVI procedures were a major cause of mortality and morbidity in the initial phase of TAVI procedures in the case of a transfemoral access path $[7,8,43]$. However, as a result of the use of smaller delivery systems and better patient selection, the rates of vascular complications could be significantly lowered [44].

Relevant arteriosclerosis, vessel course, and the diameter of the delivery system in relation to the minimum diameter of the access path are associated with vascular complications $[44,45]$.

CT makes it possible to evaluate all common access paths in one examination. The degree of calcification of the aorta and iliofemoral vessels can be reliably visualized and stenoses or the minimum vessel diameter can be determined on the basis of curved reconstructions. Eccentric plaques or thrombotic deposits in the aorta which can represent a risk of embolization during mechanical manipulation can be 


\begin{tabular}{|llll|}
\hline parameters & 64-row & & 128-row dual-source \\
\hline & 1st phase & 2nd phase & 1st phase \\
\hline scanning area & aortic root & apex of the lung-symphysis & apex of the lung - symphysis \\
\hline ECG triggering & retrospective & none & prospective (high-pitch) \\
\hline scanning direction & caudocranial & craniocaudal & craniocaudal \\
\hline collimation $(\mathrm{mm})$ & $64 \times 0.625$ & $64 \times 0.625$ & $128 \times 0.75$ \\
\hline slice thickness $(\mathrm{mm})$ & $<1$ & $<1$ & $<1$ \\
\hline tube voltage $(\mathrm{kV})$ & $100-120$ & $100-120$ & $100-120$ \\
\hline tube current, modulated $(\mathrm{mAs})$ & 300 & 300 & 300 \\
\hline $\begin{array}{l}\text { contrast medium amount }(\mathrm{ml}) / \\
\text { flow rate }(\text { seconds) }\end{array}$ & $60 / 3$ & $60 / 3$ & $60 / 3$ \\
\hline
\end{tabular}

Table 1 Sample examination protocols for 64-row and 128-row dual-source scanners. The specified scan parameters for 64-row scanners are to be modified based on the technical conditions. For example, in the case of short switching times between the scan phases, the acquisition may be able to be completed with a single contrast medium bolus.

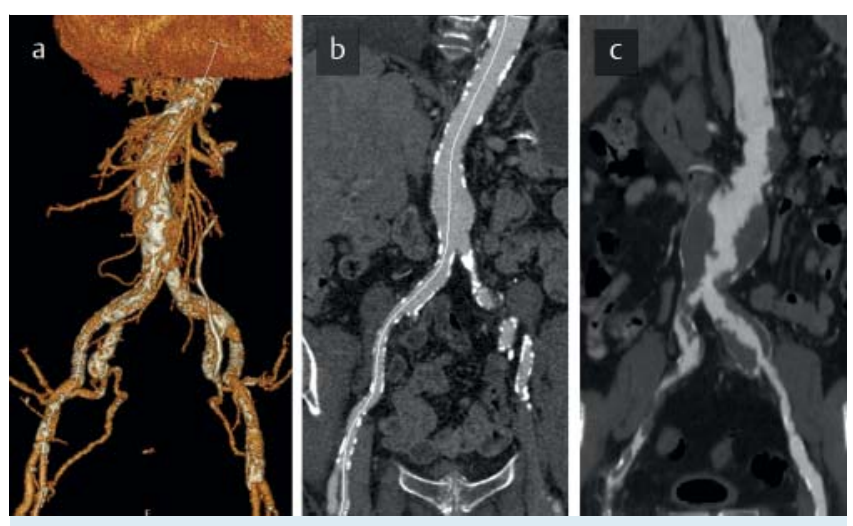

Fig. 10 a, b Three-dimensional reconstruction (volume rendering) of the abdominal aorta and curved multiplanar reformatting (cMPR) of the abdominal aorta and right iliofemoral arteries of a patient. Tortuosity of the vessel and lumen diameter are clearly visualized. c cMPR the abdominal aorta and left iliofemoral arteries of another patient with massive, partially stenotic atheromatous wall thickening.

evaluated. The vessel course and the presence of kinking can be clearly evaluated in $3 \mathrm{D}$ reconstructions ( 0 Fig. 10).

\section{Examination protocols}

The examination protocols can vary depending on the scanner technology used. To be able to plan the optimum access path, the scan should cover the entire aorta including the subclavian arteries and the iliofemoral arteries with arterial contrast enhancement. The region of the aortic root should be imaged with ECG triggering to prevent motion artifacts and to allow measurement in a defined cardiac phase, preferably in systole (30\% RR interval) [14, 23]. ECG triggering can be performed retrospectively or prospectively and should be restricted to the heart or the aortic root to minimize radiation exposure. In the case of scanners with a prospectively triggered high pitch mode, the examination can be completed with a single scan. Two acquisitions should be planned if a single source scanner is used, namely one ECG-triggered scan of the aortic root and a second scan without ECG triggering of the aorta including the subclavian arteries and the iliofemoral arteries. In the case of scanners with a short switching time between two scans, one contrast medium bolus is sufficient. Otherwise, a second bolus is needed for the second acquisition. Sample scan protocols for 64-row and 128-row dual-source units are provided in - Table 1.
Table 2 Relevant parameters.

\begin{tabular}{l} 
method \\
mode of image data acquisition \\
ECG triggering, specification of the selected cardiac phase \\
amount of contrast medium \\
findings/evaluation \\
aortic annulus: \\
- short/long diameter \\
- area/perimeter-based diameter \\
- area and perimeter \\
- fluoroscopy projection angle parallel to annulus \\
aortic valve/root: \\
- valve morphology (tricuspid/bicuspid) \\
- degree of calcification and calcification distribution of the valves \\
- max. sinus diameter \\
- diameter of the sinotubular junction \\
- diameter of the ascending aorta \\
- distance between the coronary ostia and annulus, position of the \\
coronary ostia \\
aorta and iliofemoral arteries: \\
- morphology (kinking, aneurysm, stenosis, intraluminal thrombus, \\
- degree of calcification) \\
- min. diameter of the iliofemoral vessels \\
- typical findings \\
\hline
\end{tabular}

Slice thicknesses of less than $1 \mathrm{~mm}$ are desirable for the necessary multiplanar reconstructions.

In principle, the lowest possible amount of contrast medium should be selected due to the renal insufficiency that is often present in patients prior to TAVI. Protocols with reduced amounts of contrast medium of up $50 \mathrm{ml}$ for the above-mentioned scanning area are described in the literature [46]. However, such a low amount of contrast medium can only be achieved in the case of units with a very high scanning speed.

Although the coronary arteries are always also scanned, they usually cannot be satisfactorily assessed due to the often increased heart rate in these patients caused by symptomatic AS. Lowering the heart rate with betablockers is not recommended because of the severe AS. Furthermore, the attempt to use a minimal amount of contrast medium results in iodine administration rates that are less than optimal for coronary evaluation.

\section{Findings and image documentation}

Due to the complexity of TAVI evaluation and the numerous parameters, it is recommended to use standardized findings and image documentation to record the measured 
values in order to ensure clarity. The relevant findings parameters are specified in detail in a table in the SCCT consensus document and are summarized in $\bullet$ Tab. 2 [14]. Image documentation depends on the conditions of the particular institution and can only be represented here by way of example on the basis of our own experience. In general, it is recommended to generate a $3 \mathrm{D}$ reconstruction of the aorta including the pelvic vessels for orientational evaluation of the vessel course, potential aneurysms, and the degree of calcification. The right and left pelvic axes as well as the aorta can be effectively evaluated with regard to stenosis as curved reconstructions in a radial series. Potential stenoses can be measured on most postprocessing workstations and the minimum vessel diameters can be stored as image findings. The aortic annulus measurement should start with clear documentation of the planning of the aortic annulus plane and then include short and long diameters, areabased and circumference-based diameters, area and circumference of the aortic annulus as image findings. The coronary distances and the diameter of the sinus, sinotubular junction and the ascending aorta are to be documented as additional image findings.

\section{Summary}

$\nabla$

CT is of central importance among the imaging modalities available for evaluating patients prior to TAVI. It can reliably acquire all imaging-based parameters required for adequate patient selection and procedure planning in one scan. This includes the dimensions of the aortic root including the size of the aortic annulus, the degree of calcification of the valve, and the morphology of the possible access paths. CT makes it possible to measure the aortic annulus more precisely than with 2 D TEE and to evaluate the risk for paravalvular leaks on the basis of the degree of calcification of the aortic valve. Therefore, CT is suitable as the basis for selecting prostheses and the type of implantation.

\section{Acknowledgement}

This manuscript is dedicated to Professor Bernd Hamm for his 60th birthday.

\section{References}

1 Cribier A, Eltchaninoff $H$, Bash $A$ et al. Percutaneous transcatheter implantation of an aortic valve prosthesis for calcific aortic stenosis: first human case description. Circulation 2002; 106: 3006-3008

2 Holmes DR, Mack MJ, Kaul S et al. 2012 ACCF/AATS/SCAI/STS Expert Consensus Document on Transcatheter Aortic Valve Replacement: Developed in collaboration with the American Heart Association, American Society of Echocardiography, European Association for CardioThoracic Surgery, Heart Failure Society of America, Mended Hearts, Society of Cardiovascular Anesthesiologists, Society of Cardiovascular Computed Tomography, and Society for Cardiovascular Magnetic Resonance. Ann Thorac Surg 2012; 93: 1340 - 1395

3 Nkomo VT, Gardin JM, Skelton TN et al. Burden of valvular heart diseases: a population-based study. Lancet 2006; 368: 1005-1011

4 Joint Task Force on the Management of Valvular Heart Disease of the European Society of Cardiology (ESC), European Association for Cardio-Thoracic Surgery (EACTS). Vahanian A, Alfieri O, Andreotti $F$ et al. Guidelines on the management of valvular heart disease (version 2012). Eur Heart J 2012; 33: 2451 - 2496
5 Iung B, Cachier A, Baron G et al. Decision-making in elderly patients with severe aortic stenosis: why are so many denied surgery? Eur Heart J 2005; 26: 2714-2720

6 Carabello BA, Paulus WJ. Aortic stenosis. Lancet 2009; 373: 956-966

7 Leon MB, Smith CR, Mack M et al. Transcatheter aortic-valve implantation for aortic stenosis in patients who cannot undergo surgery. N Engl J Med 2010; 363: 1597 - 1607

8 Smith CR, Leon MB, Mack MJ et al. Transcatheter versus surgical aorticvalve replacement in high-risk patients. N Engl J Med 2011; 364: $2187-2198$

9 Abdel-Wahab $M$, Zahn $R$, Horack $M$ et al. Aortic regurgitation after transcatheter aortic valve implantation: incidence and early outcome. Results from the German transcatheter aortic valve interventions registry. Heart 2011; 97: 899-906

10 Delgado V, Ewe SH, $\mathrm{Ng} \mathrm{AC}$ et al. Multimodality imaging in transcatheter aortic valve implantation: key steps to assess procedural feasibility. EuroIntervention 2010; 6: 643-652

11 Kaleschke G, Seifarth $H$, Kerckhoff $G$ et al. Imaging decision-making for transfemoral or transapical approach of transcatheter aortic valve implantation. EuroIntervention 2010; 6: G20-G27

12 Schoenhagen P, Numburi U, Halliburton SS et al. Three-dimensional imaging in the context of minimally invasive and transcatheter cardiovascular interventions using multi-detector computed tomography: from pre-operative planning to intra-operative guidance. Eur Heart J 2010; 31: $2727-2740$

13 Achenbach S, Barkhausen J, Beer $M$ et al. Consensus recommendations of the German Radiology Society (DRG), the German Cardiac Society (DGK) and the German Society for Pediatric Cardiology (DGPK) on the Use of Cardiac Imaging with Computed Tomography and Magnetic Resonance Imaging. Fortschr Röntgenstr 2012; 184: 345-368

14 Achenbach S, Delgado V, Hausleiter J et al. SCCT expert consensus document on computed tomography imaging before transcatheter aortic valve implantation (TAVI)/transcatheter aortic valve replacement (TAVR). J Cardiovasc Comput Tomogr 2012; 6: 366-380

15 Lehmkuhl LH, von Aspern K, Foldyna B et al. Comparison of aortic root measurements in patients undergoing transapical aortic valve implantation (TA-AVI) using three-dimensional rotational angiography (3DRA) and multislice computed tomography (MSCT): differences and variability. Int J Cardiovasc Imaging 2012; 29: 417-424

16 Haensig M, Holzhey DM, Lehmkuhl L et al. Experience with anatomically orientated devices for transapical aortic valve implantation. Minerva Cardioangiol 2013; 61: 33-43

17 Delgado $V, N g$ AC, Shanks $M$ et al. Transcatheter aortic valve implantation: role of multimodality cardiac imaging. Expert Rev Cardiovasc Ther 2010; 8: 113-123

18 Webb JG, Altwegg L, Masson JB et al. A new transcatheter aortic valve and percutaneous valve delivery system. J Am Coll Cardiol 2009; 53: $1855-1858$

19 Bruschi G, de Marco F, Botta L et al. Direct aortic access for transcatheter self-expanding aortic bioprosthetic valves implantation. Ann Thorac Surg 2012; 94: 497-503

20 Piazza N, de Jaegere P, Schultz C et al. Anatomy of the aortic valvar complex and its implications for transcatheter implantation of the aortic valve. Circ Cardiovasc Interv 2008; 1: 74-81

21 Anderson RH. Clinical anatomy of the aortic root. Heart 2000; 84: 670673

22 de Heer LM, Budde RP, Mali WP et al. Aortic root dimension changes during systole and diastole: evaluation with ECG-gated multidetector row computed tomography. Int J Cardiovasc Imaging 2011; 27: 1195 1204

23 Lehmkuhl L, Foldyna B, Von Aspern K et al. Inter-individual variance and cardiac cycle dependency of aortic root dimensions and shape as assessed by ECG-gated multi-slice computed tomography in patients with severe aortic stenosis prior to transcatheter aortic valve implantation: is it crucial for correct sizing? Int J Cardiovasc Imaging 2013; 29: $693-703$

24 Baumgartner $\mathrm{H}$. Hemodynamic assessment of aortic stenosis: are there still lessons to learn? J Am Coll Cardiol 2006; 47: 138-140

25 American College of Cardiology, American Heart Association Task Force on Practice Guidelines (Writing Committee to revise the 1998 guidelines for the management of patients with valvular heart disease), Society of Cardiovascular Anesthesiologists. ACC/AHA 2006 guidelines for the management of patients with valvular heart disease: a report of the American College of Cardiology/American Heart Association Task Force on Practice Guidelines (writing Committee to Revise 
the 1998 guidelines for the management of patients with valvular heart disease) developed in collaboration with the Society of Cardiovascular Anesthesiologists endorsed by the Society for Cardiovascular Angiography and Interventions and the Society of Thoracic Surgeons. J Am Coll Cardiol 2006; 48: e1 - e148

26 Vahanian A, Baumgartner H, Bax J et al. Guidelines on the management of valvular heart disease: The Task Force on the Management of Valvular Heart Disease of the European Society of Cardiology. Eur Heart J 2007; 28: 230-268

27 Tops LF, Wood DA, Delgado $V$ et al. Noninvasive evaluation of the aortic root with multislice computed tomography implications for transcatheter aortic valve replacement. JACC Cardiovasc Imaging 2008; 1 : $321-330$

28 Smíd M, Ferda J, Baxa J et al. Aortic annulus and ascending aorta: comparison of preoperative and periooperative measurement in patients with aortic stenosis. Eur J Radiol 2010; 74: $152-155$

$29 \mathrm{Ng} \mathrm{AC}$, Delgado $\mathrm{V}$, van der Kley F et al. Comparison of aortic root dimensions and geometries before and after transcatheter aortic valve implantation by 2- and 3-dimensional transesophageal echocardiography and multislice computed tomography. Circ Cardiovasc Imaging 2010; 3: $94-102$

30 Messika-Zeitoun D, Serfaty JM, Brochet E et al. Multimodal assessment of the aortic annulus diameter: implications for transcatheter aortic valve implantation. J Am Coll Cardiol 2010; 55: 186-194

31 Schultz CJ, Moelker A, Piazza N et al. Three dimensional evaluation of the aortic annulus using multislice computer tomography: are manufacturer's guidelines for sizing for percutaneous aortic valve replacement helpful? Eur Heart J 2010; 31: 849-856

32 Hamdan A, Guetta V, Konen E et al. Deformation dynamics and mechanical properties of the aortic annulus by 4-dimensional computed tomography: insights into the functional anatomy of the aortic valve complex and implications for transcatheter aortic valve therapy. J Am Coll Cardiol 2012; 59: 119-127

33 Masson JB, Kovac J, Schuler G et al. Transcatheter aortic valve implantation: review of the nature, management, and avoidance of procedural complications. JACC Cardiovasc Interv 2009; 2: 811-820

34 Généreux P, Head SJ, Van Mieghem NM et al. Clinical outcomes after transcatheter aortic valve replacement using valve academic research consortium definitions: a weighted meta-analysis of 3,519 patients from 16 studies. J Am Coll Cardiol 2012; 59: 2317-2326

35 Walther T, Dehdashtian MM, Khanna R et al. Trans-catheter valve-invalve implantation: in vitro hydrodynamic performance of the SAPIEN +cloth trans-catheter heart valve in the Carpentier-Edwards Perimount valves. Eur J Cardiothorac Surg 2011; 40: 1120-1126

36 Haensig M, Lehmkuhl L, Rastan AJ et al. Aortic valve calcium scoring is a predictor of significant paravalvular aortic insufficiency in transapicalaortic valve implantation. Eur J Cardiothorac Surg 2012; 41: 1234 1240; discussion 1240-1241

37 Delgado $\mathrm{V}, \mathrm{Ng} A C$, van de Veire $\mathrm{NR}$ et al. Transcatheter aortic valve implantation: role of multi-detector row computed tomography to evaluate prosthesis positioning and deployment in relation to valve function. Eur Heart J 2010; 31: 1114-1123

38 John D, Buellesfeld L, Yuecel S et al. Correlation of Device landing zone calcification and acute procedural success in patients undergoing transcatheter aortic valve implantations with the self-expanding Core-Valve prosthesis. JACC Cardiovasc Interv 2010; 3: 233-243

39 Agatston AS, Janowitz WR, Hildner FJ et al. Quantification of coronary artery calcium using ultrafast computed tomography. J Am Coll Cardiol 1990; 15: 827-832

40 Tzikas A, Schultz C, Van Mieghem NM et al. Optimal projection estimation for transcatheter aortic valve implantation based on contrast-aortography: validation of a Prototype Software. Catheter Cardiovasc Interv 2010; 76: $602-607$

41 Gurvitch R, Wood DA, Leipsic J et al. Multislice computed tomography for prediction of optimal angiographic deployment projections during transcatheter aortic valve implantation. JACC Cardiovasc Interv 2010; 3: $1157-1165$

42 Kurra V, Kapadia SR, Tuzcu EM et al. Pre-procedural imaging of aortic root orientation and dimensions: comparison between X-ray angiographic planar imaging and 3-dimensional multidetector row computed tomography. JACC Cardiovasc Interv 2010; 3: 105-113

43 Généreux P, Head SJ, Van Mieghem NM et al. Clinical outcomes after transcatheter aortic valve replacement using valve academic research consortium definitions: a weighted meta-analysis of 3,519 patients from 16 studies. J Am Coll Cardiol 2012; 59: 2317-2326

44 Toggweiler S, Gurvitch R, Leipsic J et al. Percutaneous aortic valve replacement: vascular outcomes with a fully percutaneous procedure. J Am Coll Cardiol 2012; 59: 113-118

45 Hayashida $K$, Lefèvre $T$, Chevalier $B$ et al. Transfemoral aortic valve implantation new criteria to predict vascular complications. JACC Cardiovasc Interv 2011; 4: $851-858$

46 Wuest W, Anders K, Schuhbaeck A et al. Dual source multidetector CTangiography before Transcatheter Aortic Valve Implantation (TAVI) using a high-pitch spiral acquisition mode. Eur Radiol 2012; 22: 51 58 